\title{
Oscillatory and nonoscillatory solutions of neutral differential equations
}

\author{
by Satoshi Tanaka (Matsuyama)
}

\begin{abstract}
Neutral differential equations are studied. Sufficient conditions are obtained to have oscillatory solutions or nonoscillatory solutions. For the existence of solutions, the Schauder-Tikhonov fixed point theorem is used.
\end{abstract}

1. Introduction. In this paper we consider the neutral differential equation

$$
\frac{d^{n}}{d t^{n}}[x(t)+\lambda x(t-\tau)]+f(t, x(g(t)))=0 .
$$

Throughout, the following conditions (H1)-(H3) are assumed:

$$
\begin{array}{ll}
\text { (H1) } & n \in \mathbb{N}, \lambda>0 \text { and } \tau>0 ; \\
\text { (H2) } & g \in C\left[t_{0}, \infty\right) \text { and } \lim _{t \rightarrow \infty} g(t)=\infty ;
\end{array}
$$

(H3) $f \in C\left(\left[t_{0}, \infty\right) \times \mathbb{R}\right)$ and there exists $F \in C\left(\left[t_{0}, \infty\right) \times[0, \infty)\right)$ such that $F(t, u)$ is nondecreasing in $u \in[0, \infty)$ for each fixed $t \geq t_{0}$ and satisfies

$$
|f(t, u)| \leq F(t,|u|), \quad(t, u) \in\left[t_{0}, \infty\right) \times \mathbb{R} .
$$

By a solution of (1.1) we mean a function $x(t)$ which is continuous and satisfies (1.1) on $\left[t_{x}, \infty\right)$ for some $t_{x} \geq t_{0}$. Therefore, if $x(t)$ is a solution of (1.1), then $x(t)+\lambda x(t-\tau)$ is $n$ times continuously differentiable on $\left[t_{x}, \infty\right)$. Note that, in general, $x(t)$ itself is not continuously differentiable.

A solution of (1.1) is called oscillatory if it has arbitrarily large zeros; otherwise it is called nonoscillatory. This means that a solution $x(t)$ is oscillatory if and only if there is a sequence $\left\{t_{i}\right\}_{i=1}^{\infty}$ such that $t_{i} \rightarrow \infty$ as $i \rightarrow \infty$ and $x\left(t_{i}\right)=0(i=1,2, \ldots)$, and a solution $x(t)$ is nonoscillatory if and only if $x(t)$ is either eventually positive or eventually negative.

2000 Mathematics Subject Classification: Primary 34K11.

Key words and phrases: oscillatory solution, nonoscillatory solution, neutral differential equation. 
There has been much current interest in the existence of oscillatory solutions and nonoscillatory solutions of neutral differential equations, and many results have been obtained. For typical results, we refer to the papers $[1,5-15]$ and the monographs $[2,3]$.

Neutral differential equations find numerous applications in natural science and technology. For instance, they are frequently used for the study of distributed networks containing lossless transmission lines. See, for example, Hale [4].

Now consider the equation

$$
\frac{d^{n}}{d t^{n}}[x(t)-\lambda x(t-\tau)]+f(t, x(g(t)))=0 .
$$

Let $\omega, \omega_{-} \in C(\mathbb{R})$ satisfy $\omega(t+\tau)=-\omega(t)$ and $\omega_{-}(t+\tau)=\omega_{-}(t)$, respectively, for $t \in \mathbb{R}$. For example, $\omega(t)=\sin (\pi t / \tau)$ and $\omega_{-}(t)=\cos (2 \pi t / \tau)$ are such functions. We easily see that $\lambda^{t / \tau} \omega(t)$ and $\lambda^{t / \tau} \omega_{-}(t)$ are solutions of the unperturbed equations

$$
\frac{d^{n}}{d t^{n}}[x(t)+\lambda x(t-\tau)]=0 \quad \text { and } \quad \frac{d^{n}}{d t^{n}}[x(t)-\lambda x(t-\tau)]=0,
$$

respectively. Thus it is natural to expect that, if $f$ is small enough in some sense, equation (1.1) [resp. (1.2)] has a solution $x(t)$ which behaves like the function $\lambda^{t / \tau} \omega(t)$ [resp. $\left.\lambda^{t / \tau} \omega_{-}(t)\right]$ as $t \rightarrow \infty$. In fact, the following results have been established by Jaroš and Kusano [7].

Theorem A. Suppose that $0<\lambda \leq 1$ and that there exist constants $\mu \in(0, \lambda)$ and $a>0$ such that

$$
\int_{t_{0}}^{\infty} t^{n-1} \mu^{-t / \tau} F\left(t, a \lambda^{g(t) / \tau}\right) d t<\infty .
$$

Then

(i) for each $\omega \in C(\mathbb{R})$ such that $\omega(t+\tau)=-\omega(t)$ for $t \in \mathbb{R}$ and $\max _{t \in \mathbb{R}}|\omega(t)|<a$, equation (1.1) has a solution $x(t)$ satisfying

$$
x(t)=\lambda^{t / \tau}[\omega(t)+o(1)] \quad(t \rightarrow \infty),
$$

(ii) for each $\omega_{-} \in C(\mathbb{R})$ such that $\omega_{-}(t+\tau)=\omega_{-}(t)$ for $t \in \mathbb{R}$ and $\max _{t \in \mathbb{R}}\left|\omega_{-}(t)\right|<a$, equation (1.2) has a solution $x(t)$ satisfying

$$
x(t)=\lambda^{t / \tau}\left[\omega_{-}(t)+o(1)\right] \quad(t \rightarrow \infty) .
$$

Theorem B. Suppose that $\lambda>1$ and that there exist constants $\mu \in(1, \lambda)$ and $a>0$ such that

$$
\int_{t_{0}}^{\infty} \mu^{-t / \tau} F\left(t, a \lambda^{g^{*}(t) / \tau}\right) d t<\infty,
$$

where $g^{*}(t)=\max \{g(t), t\}$. Then (i) and (ii) of Theorem A follow. 
We note that a solution $x(t)$ satisfying (1.3) is oscillatory if $\omega(t) \not \equiv 0$, and that a solution $x(t)$ satisfying (1.4) is oscillatory or nonoscillatory according to whether the function $\omega_{-}(t)$ is oscillatory or nonoscillatory. In particular, Theorems A and B are first results concerning the existence of oscillatory solutions of nonlinear neutral differential equations.

For equation (1.2), Theorems $\mathrm{A}$ and $\mathrm{B}$ have been extended to the following results by Kitamura and Kusano [9]. (See also [5, 8, 10, 14].)

Theorem C. Let $\lambda=1$. Suppose that

$$
\int_{t_{0}}^{\infty} t^{n} F(t, a) d t<\infty \quad \text { for some } a>0 .
$$

Then, for each $\omega_{-} \in C(\mathbb{R})$ such that $\omega_{-}(t+\tau)=\omega_{-}(t)$ for $t \in \mathbb{R}$ and $\max _{t \in \mathbb{R}}\left|\omega_{-}(t)\right|<a$, equation (1.2) has a solution $x(t)$ satisfying

$$
x(t)=\omega_{-}(t)+o(1) \quad(t \rightarrow \infty) .
$$

Theorem D. Let $\lambda \neq 1$. Suppose that

$$
\int_{t_{0}}^{\infty} \lambda^{-t / \tau} F\left(t, a \lambda^{g(t) / \tau}\right) d t<\infty \quad \text { for some } a>0 .
$$

Then (ii) of Theorem A follows.

However, very little is known about extensions of Theorems A and B for equation (1.1) such as Theorems $\mathrm{C}$ and $\mathrm{D}$. In this paper we obtain the following results which improve Theorems $\mathrm{A}$ and $\mathrm{B}$ for equation (1.1).

Theorem 1.1. Let $\lambda=1$. Suppose that

$$
\int_{t_{0}}^{\infty} t^{n-1} F(t, a) d t<\infty \quad \text { for some } a>0 .
$$

Then, for each $c \in \mathbb{R}$ and $\omega \in C(\mathbb{R})$ such that $\omega(t+\tau)=-\omega(t)$ for $t \in \mathbb{R}$ and $\max _{t \in \mathbb{R}}|\omega(t)|+|c|<a$, equation (1.1) has a solution $x(t)$ satisfying

$$
x(t)=\omega(t)+c+o(1) \quad \text { as } t \rightarrow \infty .
$$

Theorem 1.2. Let $\lambda \neq 1$. Suppose that (1.5) holds. Then (i) of Theorem A follows.

REMARK 1.1. The solution obtained in Theorem 1.1 is oscillatory or nonoscillatory according to whether the function $\omega(t)+c$ is oscillatory or nonoscillatory. Since condition (1.6) is independent of the choice of the function $\omega(t)+c$, equation (1.1) has both oscillatory solutions and nonoscillatory 
solutions if (1.6) holds. For the case $\omega(t) \not \equiv 0$, the solution of (1.1) obtained in Theorem 1.2 is oscillatory.

The proof of Theorem 1.1 is given in Section 2. The proof of Theorem 1.2 is divided into the cases $0<\lambda<1$ and $\lambda>1$. These are considered in Sections 3 and 4, respectively. To prove the existence of solutions, we use the Schauder-Tikhonov fixed point theorem.

2. Proof of Theorem 1.1. Equation can be replaced by (1.1) with $\lambda=1$.

Let $T$ and $T_{*}$ be constants with $T-\tau \geq T_{*} \geq t_{0}$. We denote by $U\left[T_{*}, \infty\right)$ the set of all functions $u \in C\left[T_{*}, \infty\right)$ such that $\sum_{i=1}^{\infty}(-1)^{i+1} u(t+i \tau)$ converges for each fixed $t \in[T-\tau, \infty)$. To each $u \in U\left[T_{*}, \infty\right)$ we assign the function $\Phi u$ on $\left[T_{*}, \infty\right)$ by

$$
(\Phi u)(t)= \begin{cases}\sum_{i=1}^{\infty}(-1)^{i+1} u(t+i \tau), & t \geq T-\tau, \\ (\Phi u)(T-\tau), & t \in\left[T_{*}, T-\tau\right] .\end{cases}
$$

Then we see that

$$
(\Phi u)(t)+(\Phi u)(t-\tau)=u(t), \quad t \geq T, u \in U\left[T_{*}, \infty\right) .
$$

In fact,

$$
\begin{aligned}
(\Phi u)(t)+(\Phi u)(t-\tau) & =\sum_{i=1}^{\infty}(-1)^{i+1} u(t+i \tau)+\sum_{i=1}^{\infty}(-1)^{i+1} u(t+(i-1) \tau) \\
& =\sum_{i=1}^{\infty}(-1)^{i+1} u(t+i \tau)-\sum_{i=0}^{\infty}(-1)^{i+1} u(t+i \tau) \\
& =u(t), \quad t \geq T, u \in U\left[T_{*}, \infty\right) .
\end{aligned}
$$

Hereafter, $C\left[T_{*}, \infty\right)$ is regarded as the Fréchet space of all continuous functions on $\left[T_{*}, \infty\right)$ with the topology of uniform convergence on every compact subinterval of $\left[T_{*}, \infty\right)$ (the $C\left[T_{*}, \infty\right)$-topology).

We prepare the next proposition for the proof of Theorem 1.1.

Lemma 2.1. Let $T$ and $T_{*}$ be constants with $T-\tau \geq T_{*} \geq t_{0}$. Suppose that $\eta \in C[T-\tau, \infty)$ is such that $\eta(t) \geq 0$ for $t \geq T-\tau$ and $\lim _{t \rightarrow \infty} \eta(t)=0$ and define

$$
V=\left\{v \in U\left[T_{*}, \infty\right):|(\Phi v)(t)| \leq \eta(t), t \geq T-\tau\right\} .
$$

Then $\Phi$ maps $V$ into $C\left[T_{*}, \infty\right)$ and is continuous on $V$ in the $C\left[T_{*}, \infty\right)$ topology. 
Proof. If $v \in V$, then

$$
\begin{array}{rl}
\sup _{t \in[T-\tau, \infty)} \mid \sum_{i=p+1}^{\infty}(-1)^{i+1} & v(t+i \tau) \mid \\
& =\sup _{t \in[T-\tau, \infty)}\left|\sum_{i=1}^{\infty}(-1)^{i+1} v(t+p \tau+i \tau)\right| \\
& \leq \sup _{t \in[T-\tau, \infty)} \eta(t+p \tau) \\
& =\sup _{t \in[T+(p-1) \tau, \infty)} \eta(t), \quad p=0,1,2, \ldots,
\end{array}
$$

which means that the series $\sum_{i=1}^{\infty}(-1)^{i+1} v(t+i \tau)$ converges uniformly on $[T-\tau, \infty)$. Consequently, $\Phi v$ is continuous on $\left[T_{*}, \infty\right)$ for each $v \in V$ and $\Phi$ maps $V$ into $C\left[T_{*}, \infty\right)$.

Now we prove that $\Phi$ is continuous on $V$. It suffices to show that if $\left\{v_{j}\right\}_{j=1}^{\infty}$ is a sequence in $C\left[T_{*}, \infty\right)$ converging to $v \in C\left[T_{*}, \infty\right)$ in the $C\left[T_{*}, \infty\right)$-topology, then also $\Phi v_{j}$ converges to $\Phi v$ in this topology.

For any $\varepsilon>0$, there is an integer $p \geq 1$ such that

$$
\sup _{t \in[T+(p-1) \tau, \infty)} \eta(t)<\frac{\varepsilon}{3} .
$$

Take an arbitrary compact subinterval $I$ of $[T-\tau, \infty)$. There exists an integer $j_{0} \geq 1$ such that

$$
\sum_{i=1}^{p}\left|v_{j}(t+i \tau)-v(t+i \tau)\right|<\frac{\varepsilon}{3}, \quad t \in I, j \geq j_{0} .
$$

It follows from (2.2) and (2.3) that

$$
\begin{aligned}
&\left|\left(\Phi v_{j}\right)(t)-(\Phi v)(t)\right| \leq \sum_{i=1}^{p}\left|v_{j}(t+i \tau)-v(t+i \tau)\right| \\
&+\left|\sum_{i=p+1}^{\infty}(-1)^{i+1} v_{j}(t+i \tau)\right|+\left|\sum_{i=p+1}^{\infty}(-1)^{i+1} v(t+i \tau)\right| \\
&<\varepsilon, \quad t \in I, j \geq j_{0},
\end{aligned}
$$

which implies that $\Phi v_{j}$ converges to $\Phi v$ uniformly on $I$. In view of the fact that $(\Phi v)(t)=(\Phi v)(T-\tau)$ for $t \in\left[T_{*}, T-\tau\right]$ and $v \in V$, we conclude that $\Phi$ is continuous on $V$. The proof is complete.

Proof of Theorem 1.1. Put $\delta=a-|c|-\max _{t \in \mathbb{R}}|\omega(t)|>0$. Take a number $T \geq t_{0}$ so large that

$$
T_{*}=\min \{T-\tau, \inf \{g(t): t \geq T\}\} \geq t_{0}
$$


and

$$
\int_{T}^{\infty} s^{n-1} F(s, a) d s<\delta .
$$

Let

$$
G(t)= \begin{cases}\int_{t}^{\infty} \frac{(s-t)^{n-2}}{(n-2) !} F(s, a) d s, & n \geq 2, \\ F(t, a), & n=1,\end{cases}
$$

for $t \geq T$. Notice that

$$
\int_{t}^{\infty} G(s) d s=\int_{t}^{\infty} \frac{(s-t)^{n-1}}{(n-1) !} F(s, a) d s, \quad t \geq T .
$$

Denote by $Y$ the set of all functions $y \in C\left[T_{*}, \infty\right)$ such that

$$
y(t)=y(T) \quad \text { for } t \in\left[T_{*}, T\right], \quad|y(t)| \leq \int_{t}^{\infty} G(s) d s \quad \text { for } t \geq T
$$

and

$$
|y(t)-y(t+\tau)| \leq \int_{t}^{t+\tau} G(s) d s \quad \text { for } t \geq T .
$$

Obviously, $Y$ is a closed convex subset of $C\left[T_{*}, \infty\right)$.

Now we claim that if $y \in Y$, then

$$
\left|\sum_{i=1}^{m}(-1)^{i+1} y(t+i \tau)\right| \leq \int_{t+\tau}^{\infty} G(s) d s, \quad t \geq T-\tau
$$

for $m=1,2, \ldots$ If $m$ is odd, then

$$
\begin{aligned}
\left|\sum_{i=1}^{m}(-1)^{i+1} y(t+i \tau)\right| \\
\quad=\left|\sum_{j=1}^{(m-1) / 2}[y(t+(2 j-1) \tau)-y(t+2 j \tau)]+y(t+m \tau)\right| \\
\leq \sum_{j=1}^{(m-1) / 2} \int_{t+(2 j-1) \tau}^{t+2 j \tau} G(s) d s+\int_{t+m \tau}^{\infty} G(s) d s \\
\leq \int_{t+\tau}^{\infty} G(s) d s, \quad t \geq T-\tau, y \in Y .
\end{aligned}
$$


For the case where $m$ is even, using the equality

$$
\sum_{i=1}^{m}(-1)^{i+1} y(t+i \tau)=\sum_{j=1}^{m / 2}[y(t+(2 j-1) \tau)-y(t+2 j \tau)], \quad t \geq T-\tau,
$$

we get (2.6).

According to (2.6), if $m \geq p \geq 1$ and $t \in[T-\tau, \infty)$, then

$$
\begin{aligned}
\left|\sum_{i=p}^{m}(-1)^{i+1} y(t+i \tau)\right| & =\left|\sum_{i=1}^{m-p+1}(-1)^{i+p} y(t+(i+p-1) \tau)\right| \\
& =\left|\sum_{i=1}^{m-p+1}(-1)^{i+1} y(t+(p-1) \tau+i \tau)\right| \\
& \leq \int_{t+p \tau}^{\infty} G(s) d s \rightarrow 0 \quad \text { as } p \rightarrow \infty
\end{aligned}
$$

for each $y \in Y$. Hence, $Y \subset U\left[T_{*}, \infty\right)$. Letting $m \rightarrow \infty$ in (2.6), we obtain

$$
|(\Phi y)(t)| \leq \int_{t+\tau}^{\infty} G(s) d s, \quad t \geq T-\tau, y \in Y .
$$

Lemma 2.1 implies that $\Phi$ maps $Y$ into $C\left[T_{*}, \infty\right)$ and is continuous on $Y$. From (2.4), (2.5) and the last inequality, it follows that

$$
\lim _{t \rightarrow \infty}(\Phi y)(t)=0 \quad \text { and } \quad|(\Phi y)(t)| \leq \delta, \quad t \geq T_{*}, y \in Y .
$$

Set

$$
(\Omega y)(t)=\omega(t)+c+(-1)^{n-1}(\Phi y)(t), \quad t \geq T_{*}, y \in Y .
$$

Then we find that

$$
(\Omega y)(t)=\omega(t)+c+o(1) \quad(t \rightarrow \infty)
$$

and

$$
|(\Omega y)(t)| \leq|\omega(t)|+|c|+\delta \leq a, \quad t \geq T_{*}
$$

for each $y \in Y$.

We define the mapping $\mathcal{F}: Y \rightarrow C\left[T_{*}, \infty\right)$ as follows:

$$
(\mathcal{F} y)(t)= \begin{cases}\int_{t}^{\infty} \frac{(s-t)^{n-1}}{(n-1) !} f(s,(\Omega y)(g(s))) d s, & t \geq T, \\ (\mathcal{F} y)(T), & t \in\left[T_{*}, T\right] .\end{cases}
$$

By (H3) and (2.9), the mapping $\mathcal{F}$ is well defined. We have $\mathcal{F}(Y) \subset Y$. In 
fact, if $t \geq T$ and $y \in Y$, then

$$
|(\mathcal{F} y)(t)| \leq \int_{t}^{\infty} \frac{(s-t)^{n-1}}{(n-1)} F(s, a) d s=\int_{t}^{\infty} G(s) d s,
$$

by $(2.5)$, and

$$
\begin{aligned}
|(\mathcal{F} y)(t)-(\mathcal{F} y)(t+\tau)| & =\left|\int_{t}^{t+\tau} f(s,(\Omega y)(g(s))) d s\right| \\
& \leq \int_{t}^{t+\tau} F(s, a) d s=\int_{t}^{t+\tau} G(s) d s
\end{aligned}
$$

for $n=1$, and

$$
\begin{aligned}
|(\mathcal{F} y)(t)-(\mathcal{F} y)(t+\tau)| & =\left|\int_{t}^{t+\tau} \int_{s}^{\infty} \frac{(r-s)^{n-2}}{(n-2) !} f(r,(\Omega y)(g(r))) d r d s\right| \\
& \leq \int_{t}^{t+\tau} \int_{s}^{\infty} \frac{(r-s)^{n-2}}{(n-2) !} F(r, a) d r d s=\int_{t}^{t+\tau} G(s) d s
\end{aligned}
$$

for $n \neq 1$.

Since $\Omega$ is continuous on $Y$, the Lebesgue dominated convergence theorem shows that $\mathcal{F}$ is continuous on $Y$.

Now we claim that $\mathcal{F}(Y)$ is relatively compact. We note that $\mathcal{F}(Y)$ is uniformly bounded on every compact subinterval of $\left[T_{*}, \infty\right)$, because of $\mathcal{F}(Y) \subset Y$. By the Ascoli-Arzelà theorem, it suffices to verify that the family $\mathcal{F}(Y)$ is equicontinuous on every compact subinterval of $\left[T_{*}, \infty\right)$. Observe that

$$
\left|(\mathcal{F} y)^{\prime}(t)\right| \leq\left\{\begin{array}{ll}
F(t, a), & n=1, \\
\int_{T}^{\infty} s^{n-2} F(s, a) d s, & n \neq 1,
\end{array} \quad t \geq T, y \in Y .\right.
$$

Let $I$ be an arbitrary compact subinterval of $[T, \infty)$. Then we see that $\left\{(\mathcal{F} y)^{\prime}(t): y \in Y\right\}$ is uniformly bounded on $I$. The mean value theorem implies that $\mathcal{F}(Y)$ is equicontinuous on $I$. Since $\left|(\mathcal{F} y)\left(t_{1}\right)-(\mathcal{F} y)\left(t_{2}\right)\right|=0$ for $t_{1}, t_{2} \in\left[T_{*}, T\right]$, we conclude that $\mathcal{F}(Y)$ is equicontinuous on every compact subinterval of $\left[T_{*}, \infty\right)$. Thus $\mathcal{F}(Y)$ is relatively compact as claimed.

Consequently, we are able to apply the Schauder-Tikhonov fixed point theorem to the operator $\mathcal{F}$ and find that there exists a $\widetilde{y} \in Y$ such that $\widetilde{y}=\mathcal{F} \widetilde{y}$. Set $x(t)=(\Omega \widetilde{y})(t)$. From (2.8) it follows that $x(t)$ satisfies (1.7). By (2.7) and (2.1), we obtain

$$
\begin{aligned}
x(t)+x(t-\tau) & =\omega(t)+\omega(t-\tau)+2 c+(-1)^{n-1}[(\Phi \widetilde{y})(t)+(\Phi \widetilde{y})(t-\tau)] \\
& =2 c+(-1)^{n-1} \widetilde{y}(t), \\
& =2 c+(-1)^{n-1}(\mathcal{F} \widetilde{y})(t), \quad t \geq T .
\end{aligned}
$$


Therefore we see that

$$
\frac{d^{n}}{d t^{n}}[x(t)+x(t-\tau)]=(-1)^{n-1}(\mathcal{F} \widetilde{y})^{(n)}(t)=-f(t, x(g(t))), \quad t \geq T,
$$

so that $x(t)$ is a solution of (1.1). The proof is complete.

3. Proof of Theorem $1.2(0<\lambda<1)$. We need a few lemmas.

Let $T$ and $T_{*}$ be constants such that $T-\tau \geq T_{*} \geq t_{0}$. We denote by $S\left[T_{*}, \infty\right)$ the set of all functions $u \in C\left[T_{*}, \infty\right)$ such that the series

$$
\sum_{i=1}^{\infty}|u(t+i \tau)|
$$

converges uniformly on $[T-\tau, \infty)$. It is easy to see that $S\left[T_{*}, \infty\right) \subset U\left[T_{*}, \infty\right)$ and $\Phi$ maps $S\left[T_{*}, \infty\right)$ into $C\left[T_{*}, \infty\right)$.

Lemma 3.1. Let $T$ and $T_{*}$ be constants with $T-\tau \geq T_{*} \geq t_{0}$. Suppose that $\varphi \in S\left[T_{*}, \infty\right)$ satisfies $\varphi(t) \geq 0$ for $t \geq T$ and define

$$
W=\left\{w \in C\left[T_{*}, \infty\right):|w(t)| \leq \varphi(t), t \geq T\right\} .
$$

Then $W \subset S\left[T_{*}, \infty\right)$ and $\Phi$ is continuous on $W$ in the $C\left[T_{*}, \infty\right)$-topology.

Proof. It is clear that $W \subset S\left[T_{*}, \infty\right)$. Let $\varepsilon>0$. There is an integer $p \geq 1$ such that

$$
\sum_{i=p+1}^{\infty} \varphi(t+i \tau)<\frac{\varepsilon}{3}, \quad t \geq T-\tau .
$$

Take an arbitrary compact subinterval $I$ of $[T-\tau, \infty)$. Let $\left\{w_{j}\right\}_{j=1}^{\infty}$ be a sequence in $W$ converging to $w \in W$ in the $C\left[T_{*}, \infty\right)$-topology. There exists an integer $j_{0} \geq 1$ such that

$$
\sum_{i=1}^{p}\left|w_{j}(t+i \tau)-w(t+i \tau)\right|<\frac{\varepsilon}{3}, \quad t \in I, j \geq j_{0} .
$$

We see that

$$
\begin{aligned}
\left|\left(\Phi w_{j}\right)(t)-(\Phi w)(t)\right| \leq & \sum_{i=1}^{p}\left|w_{j}(t+i \tau)-w(t+i \tau)\right| \\
& +\sum_{i=p+1}^{\infty}\left|w_{j}(t+i \tau)\right|+\sum_{i=p+1}^{\infty}|w(t+i \tau)| \\
< & \frac{\varepsilon}{3}+2 \sum_{i=p+1}^{\infty} \varphi(t+i \tau)<\varepsilon, \quad t \in I, j \geq j_{0},
\end{aligned}
$$

implying that $\Phi w_{j}$ converges to $\Phi w$ uniformly on $I$. For $t \in\left[T_{*}, T-\tau\right]$, we have $\left|\left(\Phi w_{j}\right)(t)-(\Phi w)(t)\right|=\left|\left(\Phi w_{j}\right)(T-\tau)-(\Phi w)(T-\tau)\right|$. Therefore, $\Phi$ is continuous on $W$. 
Lemma 3.2. Let $u \in C\left[T_{*}, \infty\right)$. Then $u \in S\left[T_{*}, \infty\right)$ if and only if the series (3.1) converges for each fixed $t \in[T-\tau, \infty)$ and

$$
\lim _{t \rightarrow \infty} \sum_{i=1}^{\infty}|u(t+i \tau)|=0
$$

Proof. We note that if the series (3.1) converges for each fixed $t \in$ $[T-\tau, \infty)$, then

$$
\begin{aligned}
\sup _{t \in[T+m \tau, \infty)} \sum_{i=1}^{\infty}|u(t+i \tau)| & =\sup _{t \in[T-\tau, \infty)} \sum_{i=1}^{\infty}|u(t+(m+1) \tau+i \tau)| \\
& =\sup _{t \in[T-\tau, \infty)} \sum_{i=m+2}^{\infty}|u(t+i \tau)|
\end{aligned}
$$

for $m=1,2, \ldots$

First we prove the "only if" part. Assume that $u \in S\left[T_{*}, \infty\right)$. Clearly, the series (3.1) converges for each fixed $t \in[T-\tau, \infty)$. Letting $m \rightarrow \infty$ in (3.3), we have

$$
\lim _{m \rightarrow \infty} \sup _{t \in[T+m \tau, \infty)} \sum_{i=1}^{\infty}|u(t+i \tau)|=0,
$$

which implies (3.2).

Conversely, suppose that the series (3.1) converges for each fixed $t \in$ $[T-\tau, \infty)$ and $(3.2)$ holds. By (3.3) again, we obtain

$$
\lim _{m \rightarrow \infty} \sup _{t \in[T-\tau, \infty)} \sum_{i=m+2}^{\infty}|u(t+i \tau)|=0 .
$$

This shows that the series (3.1) converges uniformly on $[T-\tau, \infty)$. Hence, the "if" part follows.

Lemma 3.3. Let $0<\lambda<1$ and $k \in \mathbb{N} \cup\{0\}$. Suppose that $G \in C\left[t_{0}, \infty\right)$ satisfies

$$
G(t) \geq 0 \quad \text { for } t \geq t_{0} \quad \text { and } \quad \int_{t_{0}}^{\infty} \lambda^{-t / \tau} G(t) d t<\infty,
$$

and define the function $\varphi$ on $\left[t_{0}, \infty\right)$ by

$$
\varphi(t)=\lambda^{-t / \tau} \int_{t}^{\infty}(s-t)^{k} G(s) d s, \quad t \geq t_{0} .
$$

Then $\sum_{i=1}^{\infty} \varphi(t+i \tau)$ converges for each fixed $t \in\left[t_{0}-\tau, \infty\right)$ and tends to 0 as $t \rightarrow \infty$. 
Proof. Let $t \geq t_{0}-\tau$ be fixed. Observe that

$$
\begin{aligned}
\sum_{i=1}^{\infty} \varphi(t+i \tau) & =\sum_{i=1}^{\infty} \lambda^{-(t+i \tau) / \tau} \sum_{j=i}^{\infty} \int_{t+j \tau}^{t+(j+1) \tau}(s-t-i \tau)^{k} G(s) d s \\
& =\sum_{j=1}^{\infty} \int_{t+j \tau}^{t+(j+1) \tau} \sum_{i=1}^{j} \lambda^{(s-t-i \tau) / \tau}(s-t-i \tau)^{k} \lambda^{-s / \tau} G(s) d s .
\end{aligned}
$$

If $s \in[t+j \tau, t+(j+1) \tau]$, then $(j-i) \tau \leq s-t-i \tau \leq(j+1-i) \tau$. Hence

$$
\begin{aligned}
\sum_{i=1}^{j} \lambda^{(s-t-i \tau) / \tau}(s-t-i \tau)^{k} & \leq \tau^{k} \sum_{i=1}^{j} \lambda^{j-i}(j+1-i)^{k} \\
& =\tau^{k} \sum_{l=1}^{j} \lambda^{l-1} l^{k} \leq \tau^{k} K
\end{aligned}
$$

for $s \in[t+j \tau, t+(j+1) \tau], K=\sum_{i=1}^{\infty} \lambda^{i-1} i^{k}$. By (3.5) and (3.6), we obtain

$$
\sum_{i=1}^{\infty} \varphi(t+i \tau) \leq \tau^{k} K \int_{t+\tau}^{\infty} \lambda^{-s / \tau} G(s) d s .
$$

This completes the proof.

Proof of Theorem 1.2 $(0<\lambda<1)$. Let $0<\lambda<1$. Put $\delta=a-$ $\max _{t \in \mathbb{R}}|\omega(t)|>0, G(t)=F\left(t, a \lambda^{g(t) / \tau}\right)$, and

$$
\varphi(t)=\lambda^{-t / \tau} \int_{t}^{\infty}(s-t)^{n-1} G(s) d s \geq 0, \quad t \geq t_{0} .
$$

From Lemma 3.3 it follows that

$$
\eta(t) \equiv \sum_{i=1}^{\infty} \varphi(t+i \tau)
$$

converges for each fixed $t \in\left[t_{0}-\tau, \infty\right)$ and $\lim _{t \rightarrow \infty} \eta(t)=0$. Thus we can choose a number $T \geq t_{0}$ so large that $\eta(t) \leq \delta$ for $t \geq T-\tau$ and

$$
T_{*}=\min \{T-\tau, \inf \{g(t): t \geq T\}\} \geq t_{0} .
$$

Lemma 3.2 implies $\left.\varphi\right|_{\left[T_{*}, \infty\right)} \in S\left[T_{*}, \infty\right)$. Define

$$
Y=\left\{y \in C\left[T_{*}, \infty\right):|y(t)| \leq \varphi(t) \text { for } t \geq T_{*}\right\} .
$$

Then $Y$ is closed and convex. By Lemma 3.1, the mapping $\Phi$ is continuous on $Y$. Put

$$
(\Omega y)(t)=\omega(t)+(-1)^{n-1}(\Phi y)(t), \quad t \geq T_{*}, y \in Y .
$$


Since

$$
|(\Phi y)(t)| \leq \sum_{i=1}^{\infty} \varphi(t+i \tau)=\eta(t) \leq \delta, \quad t \geq T-\tau, y \in Y,
$$

we have

$$
|(\Omega y)(t)| \leq|\omega(t)|+\delta \leq a, \quad t \geq T_{*}, y \in Y,
$$

and

$$
(\Omega y)(t)=\omega(t)+o(1) \quad(t \rightarrow \infty), \quad y \in Y .
$$

To each $y \in Y$ we assign the function $\mathcal{F} y$ on $\left[T_{*}, \infty\right)$ by

$$
(\mathcal{F} y)(t)= \begin{cases}\lambda^{-t / \tau} \int_{t}^{\infty} \frac{(s-t)^{n-1}}{(n-1) !} f\left(s,(\Omega y)(g(s)) \lambda^{g(s) / \tau}\right) d s, & t \geq T, \\ (\mathcal{F} y)(T), & t \in\left[T_{*}, T\right] .\end{cases}
$$

In view of (3.8), we easily see that $\mathcal{F}$ is well defined and maps $Y$ into itself. Using the same arguments as in the proof of Theorem 1.1, we conclude that $\mathcal{F}$ is continuous and $\mathcal{F}(Y)$ is relatively compact. Application of the Schauder-Tikhonov fixed point theorem shows that there exists $\widetilde{y} \in Y$ such that $\widetilde{y}=\mathcal{F} \widetilde{y}$. Put $x(t)=(\Omega \widetilde{y})(t) \lambda^{t / \tau}$. Then we obtain

$$
\begin{aligned}
x(t)+\lambda x(t-\tau) & =(\Omega \widetilde{y})(t) \lambda^{t / \tau}+\lambda(\Omega \widetilde{y})(t-\tau) \lambda^{(t-\tau) / \tau} \\
& =\lambda^{t / \tau}[(\Omega \widetilde{y})(t-\tau)+(\Omega \widetilde{y})(t-\tau)] \\
& =\lambda^{t / \tau}\left[\omega(t)+\omega(t-\tau)+(-1)^{n-1}\{(\Phi \widetilde{y})(t)+(\Phi \widetilde{y})(t-\tau)\}\right] \\
& =(-1)^{n-1} \lambda^{t / \tau} \widetilde{y}(t)=(-1)^{n-1} \lambda^{t / \tau}(\mathcal{F} \widetilde{y})(t) \\
& =(-1)^{n-1} \int_{t}^{\infty} \frac{(s-t)^{n-1}}{(n-1) !} f(s, x(g(s))) d s, \quad t \geq T .
\end{aligned}
$$

By differentiation of the above equality, we conclude that $x(t)$ is a solution of (1.1). From (3.9) it follows that $x(t)$ satisfies (1.3). This completes the proof of Theorem 1.2 for the case $0<\lambda<1$.

4. Proof of Theorem $1.2(\lambda>1)$. First we prove two lemmas.

Lemma 4.1. Let $\lambda>1$ and $k \in \mathbb{N} \cup\{0\}$. Suppose that $G \in C\left[t_{0}, \infty\right)$ satisfies (3.4). Then

$$
\lim _{t \rightarrow \infty} \lambda^{-t / \tau} \int_{t_{0}}^{t+\tau}(t+\tau-s)^{k} G(s) d s=0
$$


Proof. It suffices to give the proof for the case $k=0$. In fact, if

$$
\lim _{t \rightarrow \infty} \lambda^{-t / \tau} \int_{t_{0}}^{t+\tau} G(s) d s=0
$$

then for $k \neq 0$ we have

$$
\begin{aligned}
\lim _{t \rightarrow \infty} \lambda^{-t / \tau} \int_{t_{0}}^{t+\tau}(t+\tau-s)^{k} G(s) d s & =\lim _{t \rightarrow \infty} \frac{d^{k}}{d t^{k}} \int_{t_{0}}^{t+\tau}(t+\tau-s)^{k} G(s) d s / \frac{d^{k}}{d t^{k}} \lambda^{t / \tau} \\
& =\lim _{t \rightarrow \infty} k !\left[\frac{\tau}{\log \lambda}\right]^{k} \lambda^{-t / \tau} \int_{t_{0}}^{t+\tau} G(s) d s=0 .
\end{aligned}
$$

Put $\psi(t)=\lambda^{-t / \tau} \int_{t_{0}}^{t} G(s) d s$. An easy computation shows that

$$
\int_{t_{0}}^{t} \psi(s) d s=\frac{\tau}{\log \lambda}\left[\int_{t_{0}}^{t} \lambda^{-s / \tau} G(s) d s-\psi(t)\right], \quad t \geq t_{0} .
$$

Then we have

$$
0 \leq \int_{t_{0}}^{t} \psi(s) d s \leq \frac{\tau}{\log \lambda} \int_{t_{0}}^{\infty} \lambda^{-s / \tau} G(s) d s, \quad t \geq t_{0},
$$

which implies that $\psi$ is integrable on $\left[t_{0}, \infty\right)$. It follows from $(4.2)$ that $l=\lim _{t \rightarrow \infty} \psi(t)$ exists and is a nonnegative finite value. Since $\psi$ is integrable on $\left[t_{0}, \infty\right)$, it is impossible that $l>0$. Consequently, (4.1) holds for the case $k=0$. This completes the proof.

Lemma 4.2. Let $\lambda>1$ and $k \in \mathbb{N} \cup\{0\}$. Suppose that $G \in C\left[t_{0}, \infty\right)$ satisfies (3.4), and define the function $\varphi$ on $\left[t_{0}, \infty\right)$ by

$$
\varphi(t)=\lambda^{-t / \tau} \int_{t_{0}}^{t}(t-s)^{k} G(s) d s, \quad t \geq t_{0} .
$$

Then $\sum_{i=1}^{\infty} \varphi(t+i \tau)$ converges for each fixed $t \in\left[t_{0}-\tau, \infty\right)$ and tends to 0 as $t \rightarrow \infty$.

Proof. Let $t \geq t_{0}-\tau$ be fixed. We observe that

$$
\begin{aligned}
\sum_{i=1}^{\infty} \varphi(t+i \tau)= & \sum_{i=1}^{\infty} \lambda^{-(t+i \tau) / \tau} \int_{t_{0}}^{t+\tau}(t+i \tau-s)^{k} G(s) d s \\
& +\sum_{i=2}^{\infty} \lambda^{-(t+i \tau) / \tau} \sum_{j=1}^{i-1} \int_{t+j \tau}^{t+(j+1) \tau}(t+i \tau-s)^{k} G(s) d s
\end{aligned}
$$




$$
\begin{aligned}
= & \lambda^{-t / \tau} \sum_{i=1}^{\infty} \lambda^{-i} \int_{t_{0}}^{t+\tau}(t+i \tau-s)^{k} G(s) d s \\
& +\sum_{j=1}^{\infty} \int_{t+j \tau}^{t+(j+1) \tau} \sum_{i=j+1}^{\infty} \lambda^{-(t+i \tau-s) / \tau}(t+i \tau-s)^{k} \lambda^{-s / \tau} G(s) d s \\
\equiv & I_{1}(t)+I_{2}(t) .
\end{aligned}
$$

We have

$$
(t+i \tau-s)^{k}=[(t+\tau-s)+(i-1) \tau]^{k} \leq 2^{k}\left[(t+\tau-s)^{k}+(i-1)^{k} \tau^{k}\right]
$$

for $s \in\left[t_{0}, t+\tau\right]$, because $(u+v)^{k} \leq 2^{k}\left(u^{k}+v^{k}\right)$ for $u \geq 0$ and $v \geq 0$. Therefore

$$
\begin{aligned}
I_{1}(t) \leq & \lambda^{-t / \tau} 2^{k} \sum_{i=1}^{\infty} \lambda^{-i} \int_{t_{0}}^{t+\tau}(t+\tau-s)^{k} G(s) d s \\
& +\lambda^{-t / \tau} 2^{k} \tau^{k} \sum_{i=1}^{\infty} \lambda^{-i}(i-1)^{k} \int_{t_{0}}^{t+\tau} G(s) d s \\
= & \frac{2^{k}}{\lambda-1} \lambda^{-t / \tau} \int_{t_{0}}^{t+\tau}(t+\tau-s)^{k} G(s) d s+L \lambda^{-t / \tau} \int_{t_{0}}^{t+\tau} G(s) d s,
\end{aligned}
$$

where $L=2^{k} \tau^{k} \sum_{i=1}^{\infty} \lambda^{-i}(i-1)^{k}$. By Lemma 4.1 we obtain $\lim _{t \rightarrow \infty} I_{1}(t)$ $=0$.

If $s \in[t+j \tau, t+(j+1) \tau]$, then $(i-j-1) \tau \leq t+i \tau-s \leq(i-j) \tau$. Thus

$$
\begin{aligned}
\sum_{i=j+1}^{\infty} \lambda^{-(t+i \tau-s) / \tau}(t+i \tau-s)^{k} & \leq \tau^{k} \sum_{i=j+1}^{\infty} \lambda^{-(i-j-1)}(i-j)^{k} \\
& =\tau^{k} \sum_{l=1}^{\infty} \lambda^{-l+1} l^{k} \equiv M
\end{aligned}
$$

for $s \in[t+j \tau, t+(j+1) \tau]$, and so

$$
I_{2}(t) \leq M \int_{t+\tau}^{\infty} \lambda^{-s / \tau} G(s) d s,
$$

which implies that $\lim _{t \rightarrow \infty} I_{2}(t)=0$. This completes the proof.

Proof of Theorem 1.2 $(\lambda>1)$. Define $\delta=a-\max _{t \in \mathbb{R}}|\omega(t)|>0, G(t)=$ $F\left(t, a \lambda^{g(t) / \tau}\right)$, and let

$$
\varphi(t)=\lambda^{-t / \tau} \int_{t_{0}}^{t}(t-s)^{n-1} G(s) d s \geq 0, \quad t \geq t_{0} .
$$


In view of Lemma 4.2 , we find that

$$
\eta(t) \equiv \sum_{i=1}^{\infty} \varphi(t+i \tau)
$$

converges for each fixed $t \in\left[t_{0}-\tau, \infty\right)$ and $\lim _{t \rightarrow \infty} \eta(t)=0$. Take $T \geq t_{0}$ such that $\eta(t) \leq \delta$ for $t \geq T-\tau$ and

$$
T_{*} \equiv \min \{T-\tau, \inf \{g(t): t \geq T\}\} \geq t_{0} .
$$

By virtue of Lemma 3.2, we have $\left.\varphi\right|_{\left[T_{*}, \infty\right)} \in S\left[T_{*}, \infty\right)$. We define the set $Y$ by (3.7). To each $y \in Y$ we assign the functions $\Omega y$ and $\mathcal{F} y$ on $\left[T_{*}, \infty\right)$ by

$$
(\Omega y)(t)=\omega(t)-(\Phi y)(t), \quad t \geq T_{*},
$$

and

$$
(\mathcal{F} y)(t)= \begin{cases}\lambda^{-t / \tau} \int_{T}^{t} \frac{(t-s)^{n-1}}{(n-1) !} f\left(s,(\Omega y)(g(s)) \lambda^{g(s) / \tau}\right) d s, & t \geq T, \\ 0, & t \in\left[T_{*}, T\right],\end{cases}
$$

respectively. By the same argument as in the proof of Theorem 1.2 for the case $0<\lambda<1$, we conclude that $\mathcal{F} \widetilde{y}=\widetilde{y}$ for some $\widetilde{y} \in Y$, and that $x(t) \equiv(\Omega \widetilde{y})(t) \lambda^{t / \tau}$ is a solution of (1.1) satisfying (1.3). This completes the proof of Theorem 1.2.

\section{References}

[1] Y. Chen, Existence of nonoscillatory solutions of nth order neutral delay differential equations, Funkcial. Ekvac. 35 (1992), 557-570.

[2] L. H. Erbe, Q. Kong and B. G. Zhang, Oscillation Theory for Functional Differential Equations, Marcel Dekker, New York, 1995.

[3] I. Györi and G. Ladas, Oscillation Theory of Delay Differential Equations, Oxford Univer. Press, 1991.

[4] J. K. Hale, Theory of Functional Differential Equations, Springer, New York, 1977.

[5] J. Jaroš, Y. Kitamura and T. Kusano, On a class of functional differential equations of neutral type, in: Recent Trends in Differential Equations, World Sci. Ser. Appl. Anal. 1, World Scientific, 1992, 317-333.

[6] J. Jaroš and T. Kusano, Oscillation theory of higher order linear functional differential equations of neutral type, Hiroshima Math. J. 18 (1988), 509-531.

[7] - - - Existence of oscillatory solutions for functional differential equations of neutral type, Acta Math. Univ. Comenian. 60 (1991), 185-194.

[8] Y. Kitamura and T. Kusano, Oscillation and asymptotic behavior of solutions of first-order functional differential equations of neutral type, Funkcial. Ekvac. 33 (1990), 325-343.

[9] - - - Existence theorems for a neutral functional differential equation whose leading part contains a difference operator of higher degree, Hiroshima Math. J. 25 (1995), 53-82. 
[10] Y. Kitamura, T. Kusano and B. S. Lalli, Existence of oscillatory and nonoscillatory solutions for a class of neutral functional differential equations, Math. Bohem. 120 (1995), 57-69.

[11] W. T. Li, Classifications and existence of nonoscillatory solutions of second order nonlinear neutral differential equations, Ann. Polon. Math. 65 (1997), 283-302.

[12] M. Naito, An asymptotic theorem for a class of nonlinear neutral differential equations, Czechoslovak Math. J. 48 (1998), 419-432.

[13] Y. Naito, Nonoscillatory solutions of neutral differential equations, Hiroshima Math. J. 20 (1990), 231-258.

[14] - Asymptotic behavior of decaying nonoscillatory solutions of neutral differential equations, Funkcial. Ekvac. 35 (1992), 95-110.

[15] S. Tanaka, Existence of positive solutions for a class of first-order neutral functional differential equations, J. Math. Anal. Appl. 229 (1999), 501-518.

Department of Mathematical Sciences

Faculty of Science

Ehime University

Matsuyama 790-8577, Japan
Present address:

Department of Liberal Arts and

Engineering Science

Hachinohe National College of Technology

Hachinohe 039-1192, Japan

E-mail: tanaka-g@hachinohe-ct.ac.jp 